\title{
An Experiment to Design an Operation and Maintenance System Integrating Apriori Association Rules for a Telecom Platform
}

\author{
Chengfan Li $\mathbb{D},{ }^{1}$ Lan Liu $\mathbb{D}^{2},{ }^{2}$ Junjuan Zhao, ${ }^{1}$ and Yuejun Liu ${ }^{1}$ \\ ${ }^{1}$ School of Computer Engineering and Science, Shanghai University, Shanghai 200444, China \\ ${ }^{2}$ School of Electronic and Electrical Engineering, Shanghai University of Engineering Science, Shanghai 201620, China \\ Correspondence should be addressed to Lan Liu; liulan@sues.edu.cn
}

Received 13 April 2021; Revised 15 August 2021; Accepted 16 September 2021; Published 30 September 2021

Academic Editor: Simone Morosi

Copyright (C) 2021 Chengfan Li et al. This is an open access article distributed under the Creative Commons Attribution License, which permits unrestricted use, distribution, and reproduction in any medium, provided the original work is properly cited.

\begin{abstract}
The operation and maintenance management of telecom platforms puts more emphasis on operation and alarm monitoring and less on business flow, and it is difficult to have a seamless connection between operation and maintenance and sales. To improve the cooperative efficiency and the informatization level of telecom platform operation and maintenance, a new operation and maintenance system integrating Apriori association rules for a telecom platform is proposed. In this paper, the Apriori algorithm is firstly used to analyse the fault correlation of the operation and maintenance system of the telecommunications platform to get the alarm message. Then, the fault risk of the operation and maintenance platform is evaluated intelligently by the system-business alarm causality model. Finally, based on the fixed end and the mobile end, the dynamic presentation of the system running state and alarm monitoring, device query and positioning, and online and offline inspection are implemented and designed, respectively. Experimental results show that the proposed operation and maintenance systems integrating Apriori association rules for the telecom platform innovate the cooperative mode between the operations and sales team, and the average time reduces to 3.9 minutes from 4.7 minutes and the risk forecasting accuracy increases from $8 \%$ to $26 \%$. The assumed method can significantly improve the operational efficiency and intelligent level of the telecom platform's system and business.
\end{abstract}

\section{Introduction}

With the booming development of mobile Internet, there are many new products and services constantly launched by telecom operators, and it strives to form their own unique advantages in the competition $[1,2]$. At present, a telecom operation pattern with the coexistence of fixed and mobile basic services has been formed based on their respective business platforms [3-6]. Different from the basic platform, the operation and maintenance of the business platform include platform hardware (system level) and business data (business level), namely, the combination of operation and maintenance. The operation and maintenance method of a traditional telecom platform not only is time-consuming and inefficient but also have too many process steps and are prone to errors [7-10]. Due to the number of operation and maintenance personnel of the telecom platform which is constantly decreasing under the background of intensifica- tion and integration and the current cooperative mode between the front end and back end of the telecom platform, there is generally a shortage of an effective coordination mechanism between operation and maintenance and sales. All these greatly limit the efficiency of operation and maintenance and intelligent management of the telecom platform.

On the one hand, with the continuous capacity expansion of key business and efficiency improvement of operation and maintenance in the telecom platform, the problems and fault alarm in the process of system operation are constantly increasing [11]. However, there is a prominent contradiction increasingly between traditional operation and maintenance means and management requirements because of the reducing gradually of back-end operation and maintenance personnel and high costs of subsequent development for network management [12-14]. On the other hand, the traditional information transmission 
methods on the telecom platform, such as paper, e-mail, and telephone, have the characteristics of long process flow, extracorporeal circulation, asymmetry between information input and output, etc. It is easy to cause errors and reduce the information construction of telecom operation and maintenance management. In addition, the knowledgebased maintenance has been introduced to the related areas, and the expert system, knowledge base system, and knowledge modelling [15-17] have been explored deeply and extensively.

As an important technology in the field of data mining, association rules can effectively mine the implicit association features in data [18-21]. In these common methods, the Apriori algorithm is one of the most basic data mining methods of association rules, which finds out the association relationship among different data via mining the datasets that frequently appear in the data. At present, the Apriori algorithm has already been successfully applied in business, network security, mobile communication, and other similar studies $[22,23]$. The operation and maintenance process of the telecom platform involves many links, complex information, and diverse types, and the correlation between different types and departments of information transmission is obvious [24]. The mining and detection of the associated information in the operation and maintenance of the telecom platform improve the efficiency and quality of the business platform maintenance and solve the collaboration between the platform operation and sale, and it has become an urgent issue to be solved in the telecom industry.

In this paper, we propose the Apriori algorithm to analyse the fault correlation and intelligently evaluate the fault risk of the telecom platform and to implement the system running status and alarm monitoring by HTML5, Ajax, and quick response (QR) code recognition. Limited by the time and length of the text, this paper mainly discusses the Apriori association rules and the conduction of a preliminary experiment to design an operation and maintenance system integrating Apriori association rules for a telecom platform. The results show that the designed telecom operation and maintenance system based on Apriori association rules in this paper can significantly shorten the time of fault determination and improve the accuracy of risk prediction, which is conducive to realizing seamless and high-speed flow of services and information and improving the efficiency of business processing.

The major contributions of this paper are as follows.

(1) We propose to operate and maintenance the telecom platform based on Apriori association rules; to some extent, it improves the cooperative efficiency and the informatization level of telecom platform operation and maintenance

(2) We propose to analyse the fault correlation of the operation and maintenance system by the Apriori algorithm to get the alarm message. Then, the system-business alarm causality model evaluates the fault risk of the operation and maintenance platform intelligently
(3) We propose to construct the system running state and alarm monitoring based on the fixed end and the mobile end and present the device query and positioning and online and offline inspection

The rest of this paper is organized as follows. Section 2 describes different related works. Section 3 shows the detailed Apriori algorithm with association rules. Sections 4 and 5 illustrate the experiments and the corresponding results and application. Section 6 presents the discussion of our paper. Section 7 gives conclusions and future works in the paper.

\section{Related Work}

Association rules, a method primarily used to mine interesting rules from large amounts of original data, are one of the main research directions of data mining in recent years, aimed at the different fields, such as university scientific research projects [25], hidden abstraction mining of text data [26], data itemset [27], and product and service fusion [28]. To some extent, these achievements extend the usage field of the Apriori algorithm. In addition, different rule sets are obtained via the data mining by Cui and then the crossselling model of telecom value-added services is constructed [29]. An enterprise financial risk analysis model based on interactive mining of association rules from financial indicators is proposed by Lin and Chen [30]. In the field of disaster monitoring, based on digital aerial images and the landslide caused by heavy rainfall event in the southwest of South Korea in 1998, the uncertainty of unlabelled features in the landslide is accomplished by t-SNE clustering and Apriori algorithms, and the results can provide the reference for the classification of missing or outdated spatial attribute information [31]. The correlation among tax data is mined by association rules and then established the identification path of tax evasion [32]. Via mining on microblog comment behaviour, a microblog recommendation model was constructed based on sentiment-weighted association rules from the above conducted association rule [33].

In the field of electricity and architecture, key inducements in power production safety accidents were extracted by the Apriori algorithm from the deep association rule [34], the association rules among entities are mined by the Apriori algorithm in view of the large number of concrete dam construction documents, and it significantly improved the intelligent and refined management level of concrete dam construction documents [35]. Correlation analysis on cigarette physical indicators was conducted by the Apriori association rules and pointed out that the weight was the key factor affecting suction resistance and total ventilation rate; it provided an effective reference for stability control of cigarette physical indicators [36]. In the field of finance, the shareholder association of companies was mined by the Apriori algorithm and provides effective reference suggestions for the industry shareholder association [37]. In the transportation field, the prior perception of railway network security was completed by the improved Apriori algorithm and expands the early warning means of railway network 
monitoring [38]. A parking space idle pattern mining method was proposed based on Hadoop platform, and the association rule is mined by MapReduce parallel computing framework, which improves the computational efficiency [39]. In the personal communications, the indicators including mobile device types, mobile phone apps of the same type, and advertising investment were analysed and mined by the Apriori algorithm and further explore the association rules among users, different types of apps, and advertising investment [40]. Therefore, for many different research fields and specific application requirements, the generality, predictive and prescriptive of the constructed model, is crucial. To some extent, this determines the application value of the constructed model. In this paper, we mainly discuss the Apriori association rules and the conduction of a preliminary experiment to design an operation and maintenance system for a telecom platform due to the time and length of the text.

In view of operation and maintenance of the telecom platform, there are not only a small number of telecom service platforms in mainland China at present but also a general lack of a comprehensive information support system with complete functions. In the implementation method of the fixed end of the integrated operation and maintenance system of the telecom platform, the B/S architecture could not only eliminate the need for users to install special client software but also facilitate the development and maintenance of the system, which is conducive to unified deployment and automatic upgrade and update [41]. At present, with the web technologies developing rapidly, the possibility of HTML5 and lightweight web to develop mobile applications is discussed $[42,43]$. The results show that the telecom platform with web technology can achieve richer system functions and a more humanized human-computer interaction interface, with strong experience and practicability. Meanwhile, in the operation monitoring of the telecom platform, it is necessary to find the correlation among various faults and quickly determine the cause of the faults, which requires the correlation analysis of a large number of alarm data. At the same time, the frequent itemsets of Boolean association rules were mined by the Apriori algorithm and then constructed the alarm system [44, 45]. Subsequently, the telecom alarm system was analysed by the association rules and then provided relevant tips for operation and maintenance personnel [46, 47].

\section{Apriori Methods with Association Rules}

The Apriori algorithm is one of the most basic methods in association rule mining, which mainly includes two parts including finding frequent itemsets and exploring association rules. The core of the Apriori algorithm is the support degree from frequent itemsets and the confidence from association rules.

Let us assume that $I=\left\{i_{1}, i_{2}, \cdots, i_{m}\right\}$ is the set of all the items and $D=\left\{T_{1}, T_{2}, \cdots, T_{m}\right\}$ is the object database. The elements that make up object $T_{i}$ are called terms, the collection of items is called an itemset, and an itemset containing $k$ items is called a $k$-itemset.
Definition 1. The support degree of itemset $A$ is the proportion of the number of objects containing $A$ in the object database $D$. Namely,

$$
S(A)=\frac{\text { number }(A)}{\text { number }(\text { all samples })}=P(A) .
$$

Definition 2. If the support degree of itemset $A$ is not less than the preset minimum support threshold (min_sup), namely, $S(A) \geq \min \_$sup, the itemset $A$ is called the frequent itemset and the frequent itemset containing $k$ items is called the frequent $k$-itemset.

Definition 3. An association rule can be represented as a logical expression $A \Rightarrow B$, whereinto $A$ and $B$ are the two nonempty subitemsets of $I$, respectively. Namely, $A \subset I, B \subset I$, $A \neq \varnothing, B \neq \varnothing$, and $A \cap B=\varnothing$. The measurement of association rules is usually expressed as the degree of support $(S)$, confidence $(C)$, and lift $(L)$.

For the $A \Rightarrow B$, the support degree is defined as the proportion of the number of $A \cup B$ contained in the object database $D$. Namely,

$$
S(A \Rightarrow B)=\frac{\text { number }(A \cup B)}{\text { number }(\text { all samples })}=P(A \cup B),
$$

Confidence is defined as the proportion of objects that contain itemset $A$ that also contains itemset $B$. Namely,

$$
C(A \Rightarrow B)=P(B \mid A)=\frac{P(A \cup B)}{P(A)}=\frac{\text { number }_{s_{-} \text {count }}(A \cup B)}{\text { number }_{s_{-} \text {count }}(A)},
$$

where number ${ }_{s_{-} \text {count }}(A \cup B)$ and number s_count $(A)$ are the number of objects $A \cup B$ in the object databases $D$ and $A$, respectively.

The lift degree is defined as the ratio of the proportion of objects in the object database $D$ that contains itemset $A$ and also contains itemset $B$ to the proportion of objects that contain itemset $B$. Namely,

$$
L(A \Rightarrow B)=\frac{P(A \cup B)}{P(A) P(B)}=\frac{P(B \mid A)}{P(B)} .
$$

Based on (4), it can be seen that (1) the correlation between itemset $A$ and itemset $B$ is negative when $L<1$. That is, the occurrence of itemset $A$ will inhibit the occurrence of itemset $B$. (2) It is independent of each other between the itemset $A$ and itemset $B$ and has no correlation with each other when $L=1$. (3) The correlation between itemset $A$ and itemset $B$ is positive when $L>1$. That is, the appearance of itemset $A$ can promote the appearance of itemset $B$, and the greater the value, the stronger the promoting effect.

The detailed flowage structure of the Apriori algorithm is illustrated in Figure 1. 


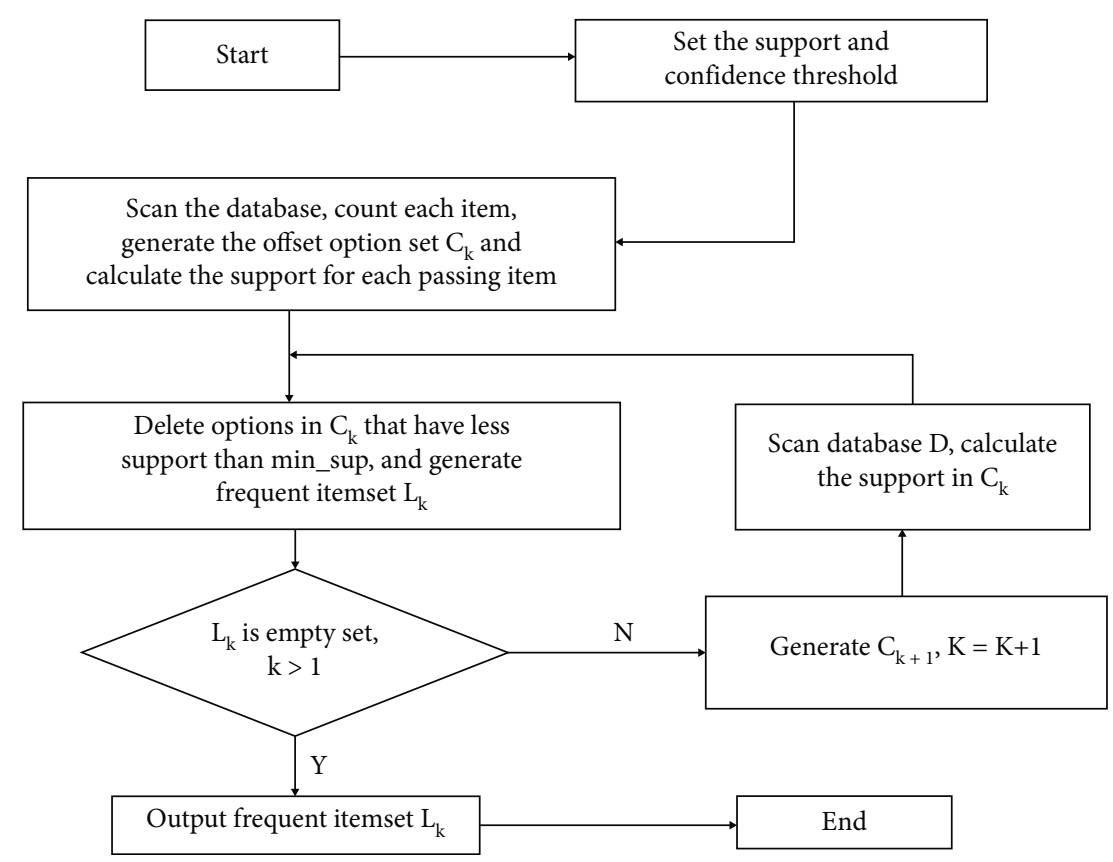

FIgURE 1: The detailed flowage structure of the Apriori algorithm.

To sum up, the Apriori algorithm mainly includes the following steps:

Step 1. Look for a frequent itemset, i.e., items whose occurrence frequency is greater than the support degree threshold.

Step 2. Generate strong association rules from a frequent itemset, and the support and confidence of these rules should be greater than the set threshold.

Step 3. Generate the expected rules using the frequent itemset obtained in Step 1, and further generate all the rules that contain the collection items.

Step 4 . In the generated rules, only those rules that satisfy a confidence level greater than the given confidence level are left. The nonfrequent itemset in the candidate set is removed, and then, all frequent itemsets are generated by the recursive method.

\section{Experiments}

4.1. Experimental System and Deployment Environment. The hardware and software environment in this experiment includes the server side of the supporting system, the data acquisition side of the platform, the fixed client, the mobile client, etc.

4.1.1. Server Side of the Supporting System. The system is implemented by Intel Xeon 4-core CPU, 8 GB RAM, 1 TB hard disk, Apache2.4 or IIS7, PHP5.5, MySQL 5.5, and x86 Server with the Windows Server 2008R2 and Linux system. In addition, the transmission rates of Internet access bandwidth or enterprise Intranet access bandwidth are greater than $1 \mathrm{Mbps}$ in the server.

4.1.2. Acquisition Side of Platform Data. The data acquisition side is deployed on the server of the business platform with the UNIX or Linux operating system and can connect with the server of the supporting system remotely by intranet and extranet.

4.1.3. Fixed-End Client. The fixed-end client is run in a web browser on a PC. In order to better be compatible with the system running state, alarm information, and interactive experience in the operation and maintenance module of the fixed end of the telecom platform, in the experiment, it is recommended to use Chrome, Firefox, and Internet Explorer with version 9.0 or above.

4.1.4. Mobile-End Client. The client is run on the apps with Android, iOS, and Windows systems. The smartphone should have the connection capability of cellular network data and Wi-Fi data, and the camera can support the scanning function of the QR code.

Taking business data collection as an example, the collected data contains the number of users, active users, calls, CRM account opening records, the call success rate, etc. When collecting the above data of the number of users, the business data acquisition script usernum.sh accessed the background Oracle database of the encrypted communication platform by the SQLPlus command and conducted data query processing on the internal data tables of multiple platforms and saved the obtained results in the record file.

Some of the core code in data collection includes the following:

On all servers where the capture scripts are deployed, the crontab is set to periodically execute the appropriate shell script. For example, on the processing interface machine, the server is used to update the system running status data every minute and the platform business data every 5 minutes. 


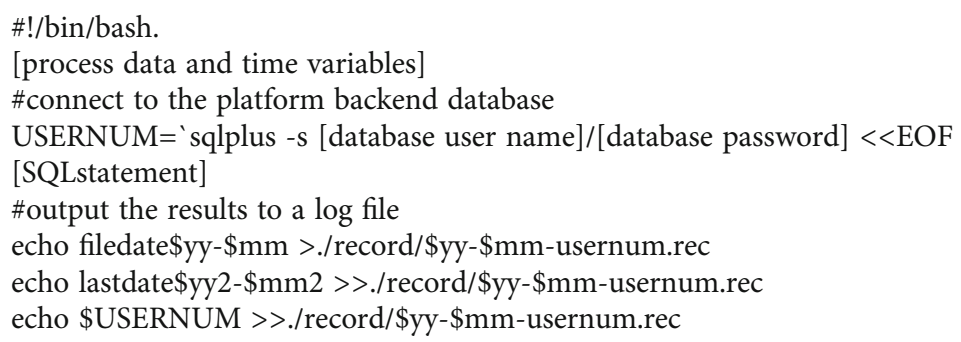

Algorithm 1: Some of the core code in data collection.

*****./report/sysmonitor/report_sysmonitor.sh \&

$* / 5{ }^{*} 1{ }^{* *} . /$ report/report_business.sh \&

Algorithm 2: Some of the core code in planning tasks.

\subsection{Experimental Details}

4.2.1. Fault Correlation Mining. In order to reduce the number of itemsets, in this paper, the alarm snapshot stored in the database at the same time interval when the alarm occurs is taken as the data source, that is, the alarm set at the same time. Correspondingly, in the experiment, the most recent 100 alarm snapshots with alarms to be calculated were selected as the original data $(D)$.

Candidate itemset 1 (i.e., $C_{1}$ ) is obtained by scanning the original data and further calculating the number of each alarm in the original data. The candidate itemset includes the support degree of each itemset. Frequent itemset 1 (i.e., $L_{1}$ ) was generated by comparing the candidate itemset, and then, the minimum support (MinSup) was set as $10 \%$. That is, the itemset with the support degree less than $10 \%$ in the candidate itemset was removed, and finally, $L_{1}$ was got.

In the next, candidate itemset 2 (i.e., $C_{2}$ ) was generated by joining and pruning operations on $L_{1}$. In the process of computing, the join operation is to lexicographically join the itemset in $L_{1}$ to $C_{2}$, and the pruning operation is to delete the itemset containing the infrequent subset after joining. Frequent itemset 2 (i.e., $L_{2}$ ) was generated by comparing $C_{2}$, and the minimum support (MinSup) was set at $10 \%$. That is, the itemset with the support less than $10 \%$ in $C_{2}$ was removed to get $L_{2}$. Meanwhile, item-associated alarm set 2 (i.e., $A_{2}$ ) was obtained via comparing $L_{2}$, and the minimum support (MinSup) is set to $50 \%$. That is, there is half of the alarm snapshots in the original data appearing in itemset $A_{2}$.

Similarly, candidate itemset 3 (i.e., $C_{3}$ ) is generated by joining and pruning operations on $L_{2}$, and then, frequent itemset 3 (i.e., $L_{3}$ ) is generated by comparing $C_{3}$; finally, item correlation alarm set $3\left(A_{3}\right)$ is obtained after comparison of $L_{3}$, and so on until the new frequent sets was no longer generate to it. In the method, the MinSup for comparing and generating frequent sets $L_{K}$ is set to $10 \%$, while the MinSup for comparing and generating associated alarm sets is differ-
TABLE 1: Relationship between the MinSup and the number of items in the itemset.

\begin{tabular}{lcc}
\hline Number of items $(K)$ & MinSup $\left(L_{k}\right)$ & MinSup $\left(A_{k}\right)$ \\
\hline 1 & $10 \%$ & $/$ \\
2 & $10 \%$ & $50 \%$ \\
3 & $10 \%$ & $35 \%$ \\
4 & $10 \%$ & $20 \%$ \\
$>4$ & $10 \%$ & $10 \%$ \\
\hline
\end{tabular}

ent. The relationship between the MinSup and the number of items in the itemset is shown in Table 1.

Figure 2 shows the alarm calculation process based on the Apriori algorithm. Candidate itemset 1 is generated by scanning the original data $D$, and the frequent itemset $K$ is generated by comparing the frequent itemset. And then, the candidate itemset $K+1$ is generated further by connecting and pruning the frequent itemset. Finally, the management alarm sets $A_{2}$ and $A_{3}$ are generated by comparing $L_{2}$ and $L_{3}$, respectively. The general collection of $A_{2}$ and $A_{3}$ (i.e., $\{A, C\}$ and $\{A, B, C\}$ ) is the relevant alarm set, namely, the alarm set of all items obtained from the original data $D$.

4.2.2. Fault Risk Assessment. The fault alarm types of the telecom platform usual include system alarm and service alarm. The former is caused by the abnormal state of system hardware and software, while the latter is caused by the abnormal business indicators of the platform.

In the experiment, the key to the fault risk assessment model based on system-business alarm causality lies in the potential causality between system faults and business alarms. That is, in the related alarm set obtained by the Apriori algorithm, to some extent, the service alarm is regarded as being caused by the system alarm in the same itemset.

In terms of the proposed model, the number and level of the associated business alarms together determine the risk level of the alarm for a single system alarm.

To quantify the alarm risk of the system, in the experiment, the fault risk is evaluated by the risk degree score model, as shown in

$$
R_{\Sigma}=\sum_{i=0}^{n} R_{i}
$$




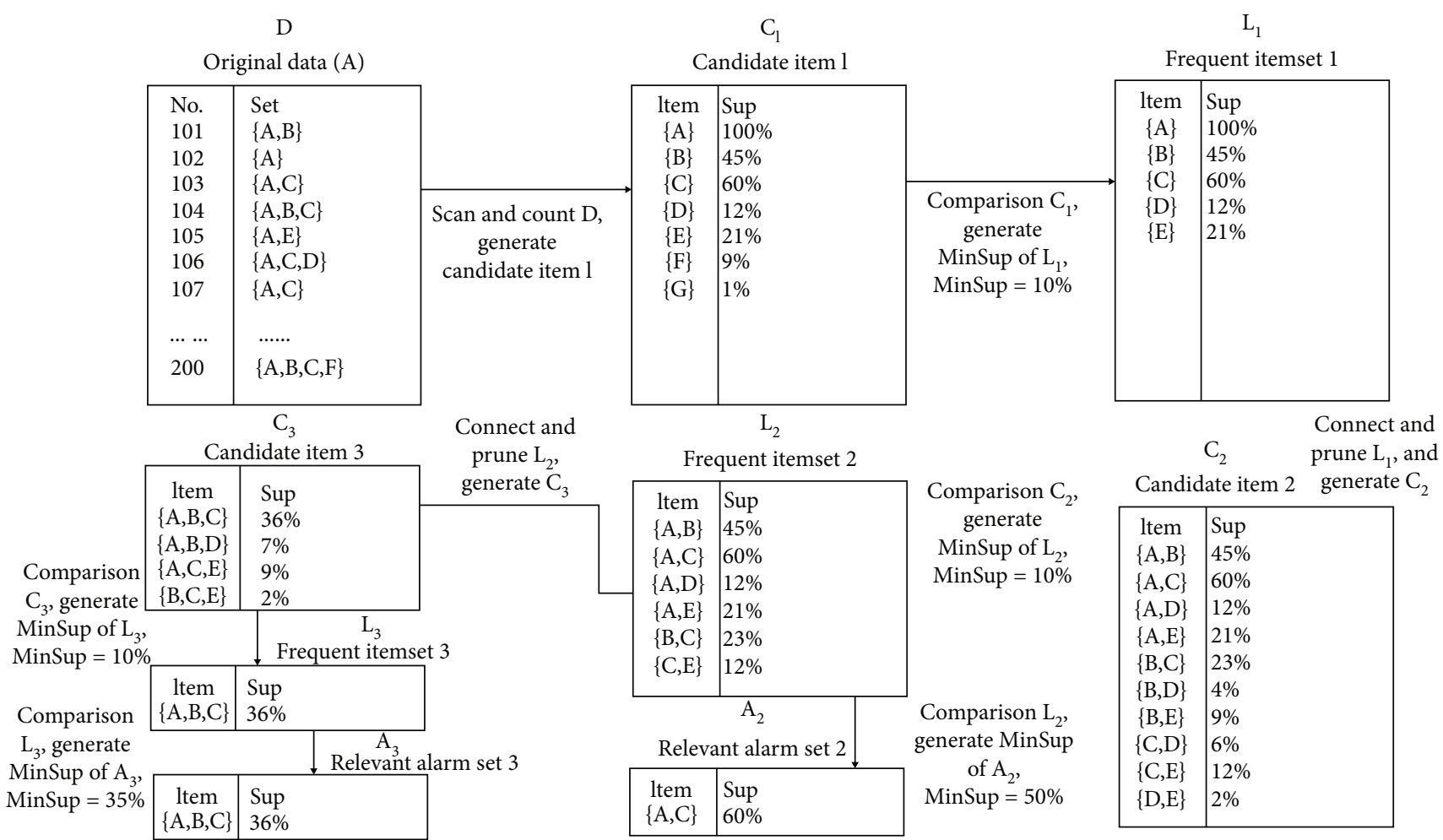

Figure 2: Alarm calculation process based on the Apriori algorithm.

where $R_{i}$ is the score of business alarm level related to the system alarm, $N$ is the total number of related business alarm, and $R_{\Sigma}$ is the risk score of system alarm.

Since the score of alarm risk varies with the number of items, the different alarm level scores are designed as equation (5). And the alarm level score of different numbers of the items is shown in Table 2.

As shown in Table 2, there are three types of alarm level including severe, serious, and general with the number of items. For the same number of item value, the alarm level score is not exactly the same. For example, when the number of items reaches 4 , the same alarm level score means the different alarm level.

Since the itemset $\{A, B, C\}$ of the associated alarm set to which $A$ and $B$ belong is a 3 -item associated alarm itemset (i.e., $K=3$ ), $R_{i}=3, n=1$, and then $R_{\Sigma}=3$, we conclude that the risk assessment level corresponding to alarm $A$ is medium, as shown in Table 3.

In Table 3, the operation and maintenance system integrating the Apriori algorithm for the telecom platform proposed in this paper has the preliminary function of alarm and alarm risk assessment, which helps to improve the fault judgment and response efficiency of the operation and maintenance of the telecom platform.

4.2.3. Monitoring Visualization of System Operation. Figure 3 shows the updating process of web page information based on Ajax and HTML technologies.

As shown in Figure 3, the program of a web front end regularly calls a jQuery function without the human intervention and accesses the operation and maintenance module
TABle 2: Different types of business alarm level.

\begin{tabular}{lcc}
\hline Number of items $(K)$ & Alarm level & Alarm level score $\left(R_{i}\right)$ \\
\hline \multirow{3}{*}{2} & Severe & 5 \\
& Serious & 3 \\
& General & 1 \\
\hline \multirow{3}{*}{3} & Severe & 3 \\
& Serious & 2 \\
& General & 1 \\
\hline \multirow{3}{*}{$\geq 4$} & Severe & 2 \\
& Serious & 1 \\
& General & 1 \\
\hline
\end{tabular}

TABLE 3: Different risk assessment levels.

\begin{tabular}{lc}
\hline$R_{\Sigma}$ & Risk assessment level \\
\hline$\geq 5$ & High \\
$3 \leq R_{\Sigma}<5$ & Medium \\
$1 \leq R_{\Sigma}<3$ & Low \\
$<1$ & Very low \\
\hline
\end{tabular}

of the web back end in the manner of HttpRequest. Via accessing the system running state and alarm information table of the system database, the web back end obtains data, and then, the assembled JSON format data is returned to the web front end by HTTP. After inputting JSON data, the 


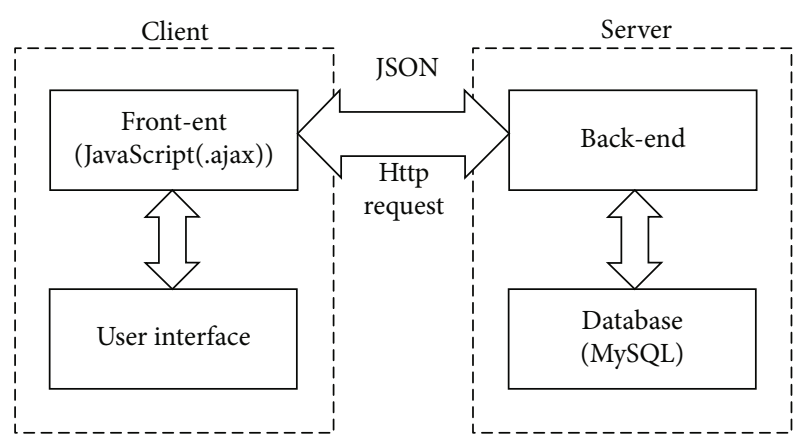

FIgURE 3: Updating process of web page information.

jQuery function in the web front end is used to identify and update HTML elements of the user interface (UI).

Some of the core code in function calls and data access includes the following:

4.2.4. Inspection Report and Mobile End. In the experiment, the inspection report of the abnormal situation is accomplished by the method of combining the fixed end and mobile end, which can scan the QR code of the equipment in the machine room with mobile phones. In the method, the key to realize the mobile-end inspection and reporting process is the identification and location of the QR code for equipment. That is, after the user scans the QR code of the device with the mobile app, the telecom platform pushes the XML message to the back end of the mobile by HTTP, and the format of the XML message is the following:

When the above XML message is received at the back end of the mobile, the XML message is parsed by the simplexml:load_string() function, and then, the link and page to report the inspection information of the room and equipment are pushed to the user.

\section{Results and Application}

The detailed software structure and implementation process are shown in Figure 4.

As shown in Figure 4, the whole software structure contains a user interaction layer, business logic layer, operation environment layer, and basic resource layer. There are fixedend UI and mobile-end UI in the operation and maintenance system.

5.1. Monitoring System of Operation and Maintenance. In the operation and maintenance of the telecom platform, the operation monitoring page of the fixed-end platform displays the running state and alarm overview of the platform, and the main index information is displayed by switching of the tab page. Meanwhile, the mobile end binds customer information and displays system running status and alarm overview information. There is an alarm message produced in line with the push standards, the mobile end can also receive the fault push message and display the relevant alarm and risk assessment information. In the process of pushing alarm message, the mobile end is mainly to scan the QR code of equipment and report the inspection information, while the fixed end in a machine room is mainly to carry out inspection records, data screening, and inspection of the equipment.

Figure 5 shows the operation monitoring interface of the telecom platform in this paper.

As shown in Figure 5, the operation and maintenance staff can monitor the disk space of the system, such as network element IP, results, and pass, whereinto the XX.XX.XX.XX. indicates the IP address that hides specific information.

Figure 6 shows the inspection reporting interface of the telecom platform based on the mobile end in this paper.

5.2. Auxiliary System of Operation and Maintenance. In the auxiliary system of operation and maintenance, the management page of monthly report in the fixed end can generate and download the monthly report; the management page of the knowledge base can view and search the knowledge experience classification. Meanwhile, the mobile end can share the experience and query the knowledge base.

Figure 7 shows the auxiliary system interface of the operation and maintenance in the telecom platform in this paper.

Figure 8 shows the monthly report generated by the auxiliary system of operation and maintenance in this paper.

Figure 9 shows the management interface of the knowledge base by the auxiliary system of the operation and maintenance in this paper.

As can be seen from Figures 8 and 9, the knowledge and experience sharing and the query of the knowledge base can be carried out normally on mobile terminals; the management page of the knowledge base in the fixed terminal platform can be smoothly classified, viewed, and searched for knowledge and experience.

5.3. Business Management and Support System. In the business management and support system, the statistics page of fixed-end business data shows the statistical charts of user development and call situation in the last month. The management page of the support order shows the view, feedback, statement, and other operations. Users can create, feedback, settle, and evaluate the service support order on the mobile end.

By taking the test data as an example, Figure 10 shows the statistical interface of business data in the business management and support system in this paper.

As shown in Figure 10, the business management and support system clearly displays the monthly business statistics (Figure 10(a)a) and change trend (Figure 10(b)) of the whole year in 2020 .

5.4. Management System. In the management system, the administrator can enter the back end of the system module. The user account can create, modify, and delete users in the management page. In the management page of the device, the device list can be displayed, and further create, modify, and delete the device and generate the QR code. Figure 11 shows the management interface of the system user.

As shown in Figure 11, in the management page of the fixed end, the administrator can edit, delete, review, and 
dataread_alarmlist $=$ function ()\{

$\$ . \operatorname{ajax}(\{$

url: "./json/dataread_alarmlist.php",

cache: false,

dataType : "json",

data: \{

\} ,

ifModified:true,

success: function(data)\{

\}

setTimeout(dataread_alarmlist,60000);

Algorithm 3: Some of the core code in function calls and data access.

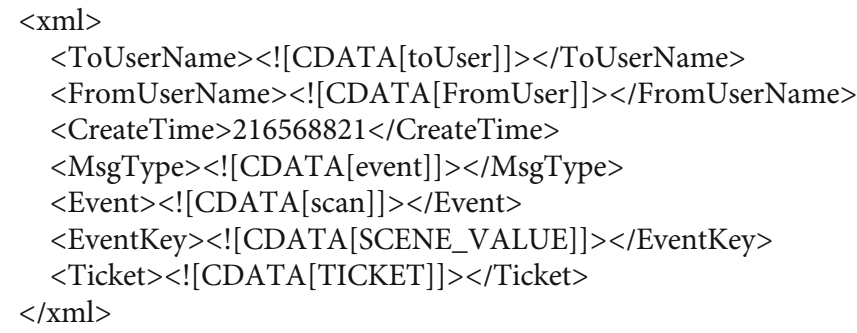

Algorithm 4: The format of the XML message by HTTP.

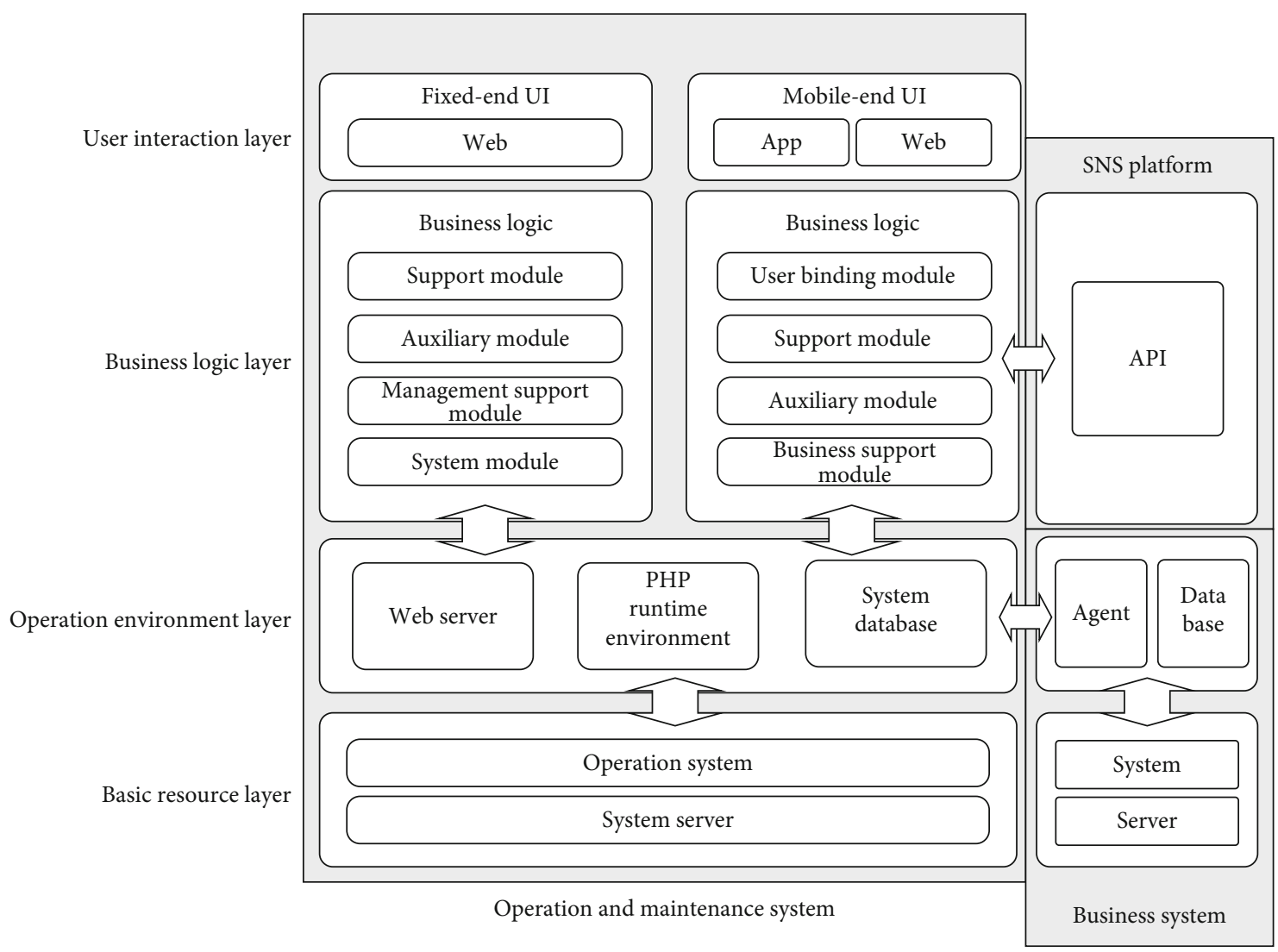

FIGURE 4: Software structure and implementation process. 


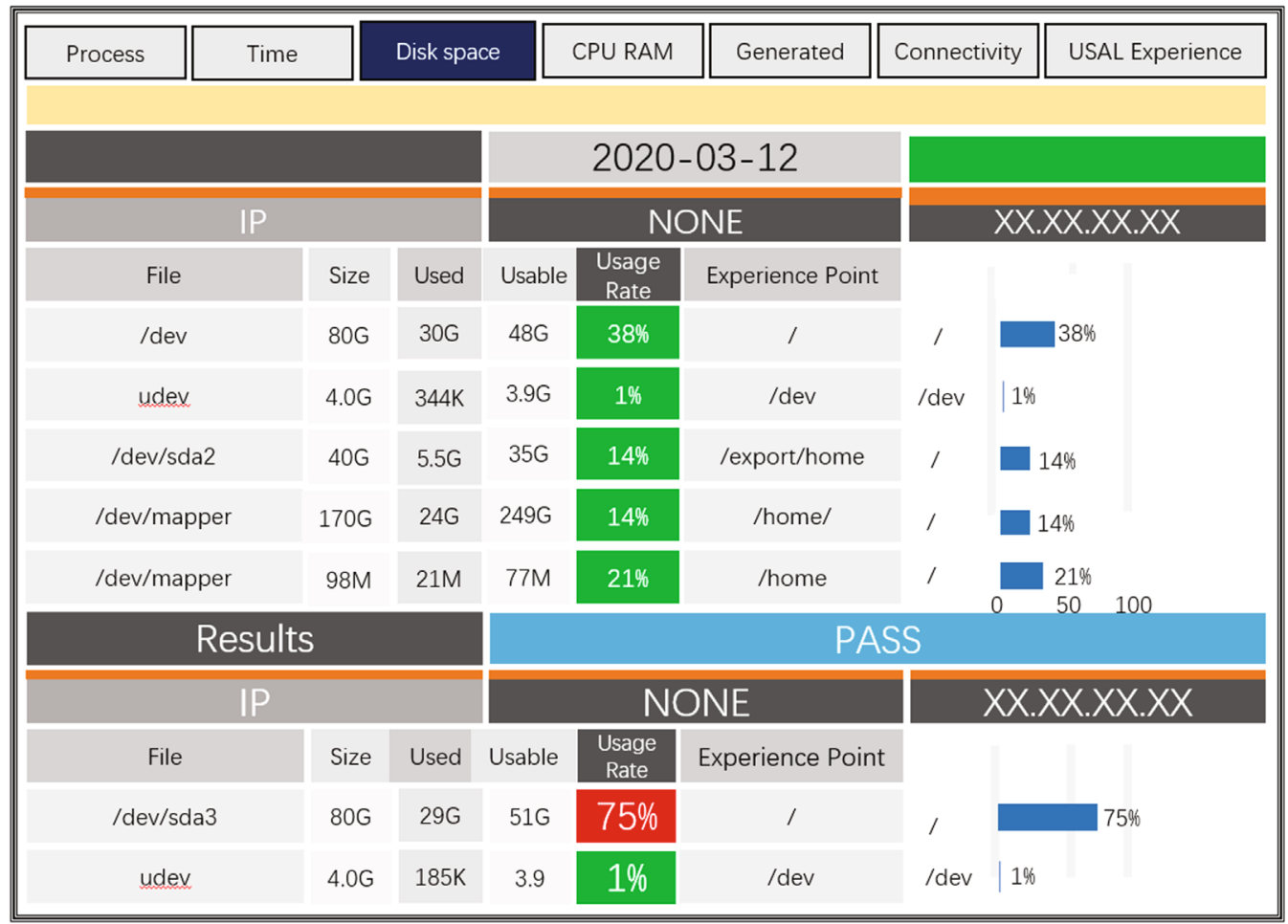

Figure 5: Operation monitoring interface of the telecom platform.

\section{Integrated Operation and Maintenance System}

\section{Dr CF LI, Hello ! \\ Unit Type: SMC-1}

\section{Type: Application Server}

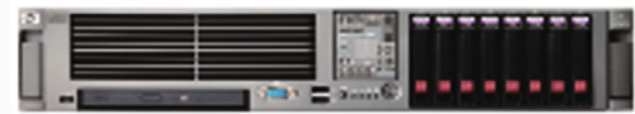

Address: B12-3011-3-1

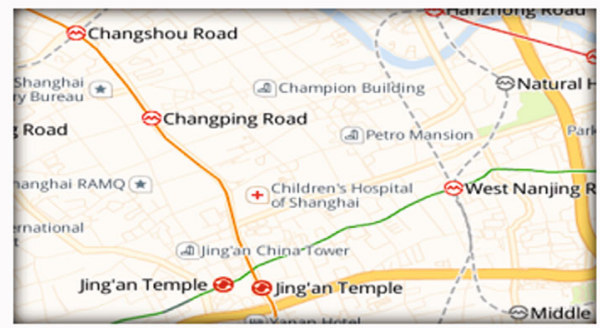

Exception Type Server Failure $\quad \mathbf{v}$

\begin{tabular}{l|l|} 
Description & $\begin{array}{l}\text { Trouble light bright } \\
\text { in Disk } 1\end{array}$ \\
\hline
\end{tabular}

FIGURE 6: Inspection reporting interface based on the mobile end. delete the basic information displayed on the main interface and delete and modify the operation log. In the management page of the mobile end, the administrator can set the menu of the mobile-end official account and modify the level of fault information push in the mobile end.

\section{Discussions}

6.1. Comparison with the Traditional Network Management Platform. Compared with the traditional network management platform, the proposed operation and maintenance system integrating Apriori association rules for the telecom platform in this paper has many benefits. It breaks through the limitations of the traditional network management system including structure, function, and cooperation mode of users.

6.1.1. Constructing the System Architecture Based on the Fusion Model of the Fixed End and Mobile End. In this system, it not only enables the operation and maintenance team to check the platform running status and alarm information by the mobile phone anytime and anywhere but also then reports the abnormal situation of equipment in real time in the machine room and receives and pushes the fault information in time. In addition, it also enables the sales team to collaborate closely with the operation and maintenance team anytime and anywhere to get timely technical support. To some extent, it overcomes the limitation that the traditional network management system can only be used in a fixed environment. 


\begin{tabular}{|c|c|c|c|c|c|}
\hline \multicolumn{6}{|l|}{ - Select time } \\
\hline Year/Month & Mar-2020 & $v$ & \multicolumn{2}{|c|}{ Submission } & \\
\hline \multicolumn{6}{|l|}{ - Generated Report } \\
\hline Year/Month & File Name/Download & & Delete & User & Created Data \\
\hline Mar-2020 & $\mathrm{Kmc} \cdots$ & & [Del] & admin & 2020-03-12 09:12:48 \\
\hline
\end{tabular}

FIgURE 7: Auxiliary system interface of the operation and maintenance.

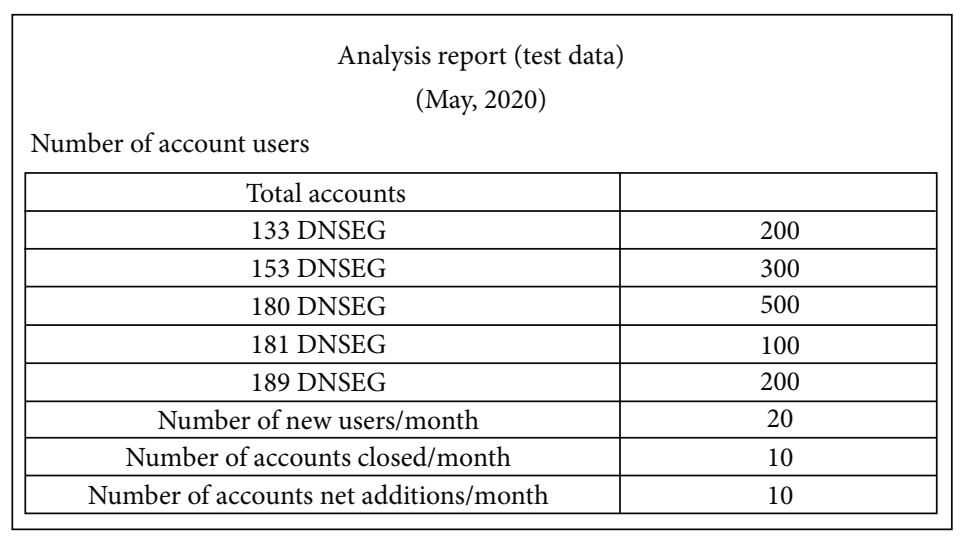

FIGURE 8: Monthly report generated by the auxiliary system.

\begin{tabular}{|c|c|c|c|c|}
\hline Content & Platform-end Interaction & Platform-CRM Interaction & Information List & \\
\hline Materials & \multirow{2}{*}{$\begin{array}{l}\text { Materials } \\
\text { Business Type }\end{array}$} & & & \\
\hline $\begin{array}{c}\text { Interface } \\
\text { Description }\end{array}$ & & & & \\
\hline \multirow{6}{*}{$\begin{array}{l}\text { Main Data } \\
\text { Reference }\end{array}$} & No. & Description & & \\
\hline & 20 & \multicolumn{2}{|c|}{ account opening / installation } & \\
\hline & 1 & \multicolumn{2}{|l|}{ modify the number } & \\
\hline & 9 & \multicolumn{2}{|c|}{ modify the ancillary products } & \\
\hline & 66 & \multicolumn{2}{|l|}{ fill in the card } & \\
\hline & 22 & \multicolumn{2}{|l|}{ modify the terminal } & \\
\hline
\end{tabular}

FIGURE 9: Management interface of the knowledge base.

6.1.2. Realizing the Comprehensive Coverage of System Operation and Maintenance and Business Support Functions. In this system, it mainly includes operation and maintenance support, auxiliary system of operation and maintenance, business management and support, and other functions. In view of the operation flow, it covers the data and business process of the whole telecom platform, such as daily monitoring and fault processing, auxiliary system, business data analysis, and team collaboration of the front end and back end. It overcomes the limitation that the tradi- tional network management system can only monitor the system running state and alarm.

6.1.3. Building the Collaborative Mode of Comprehensive Support for the Telecom Platform. Via the development of the mobile end, in this system, it connects the platform operation and sales support team together, initially realizes the cooperation between knowledge experience sharing and business support, and promotes the working efficiency of the front end and back end in the telecom platform. It 


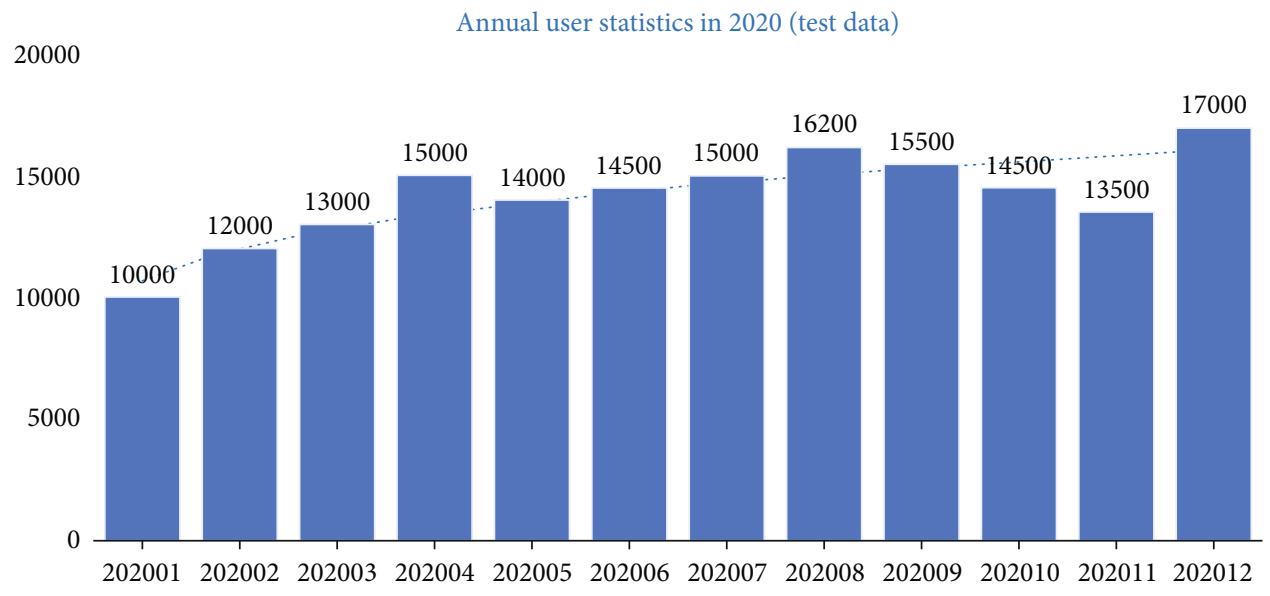

(a)

20000

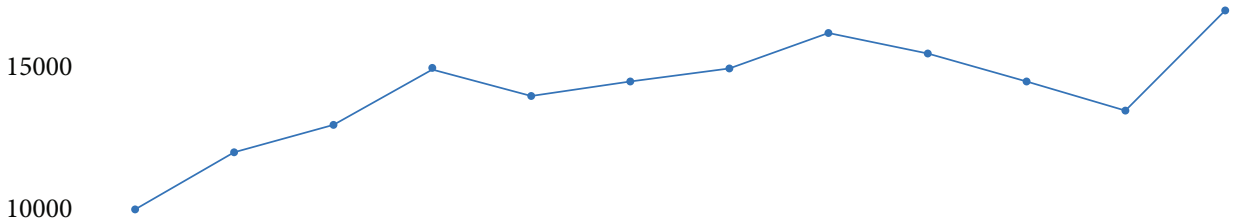

5000

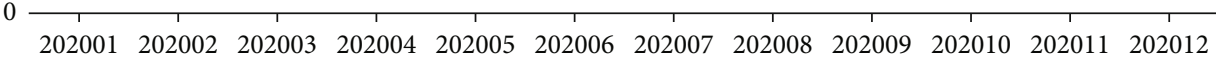

(b)

Figure 10: Statistical interface of business data (a) and the corresponding line chart (b).

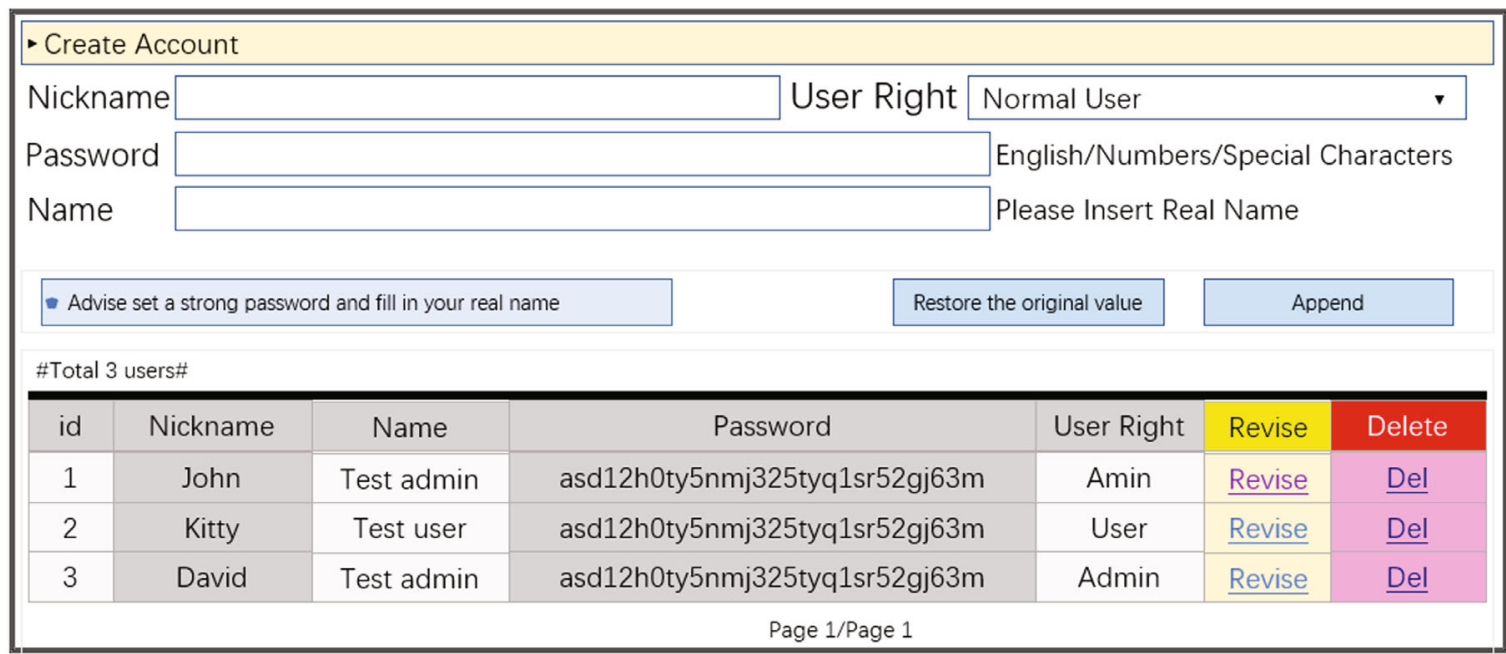

FIGURE 11: Management interface of the system user. 


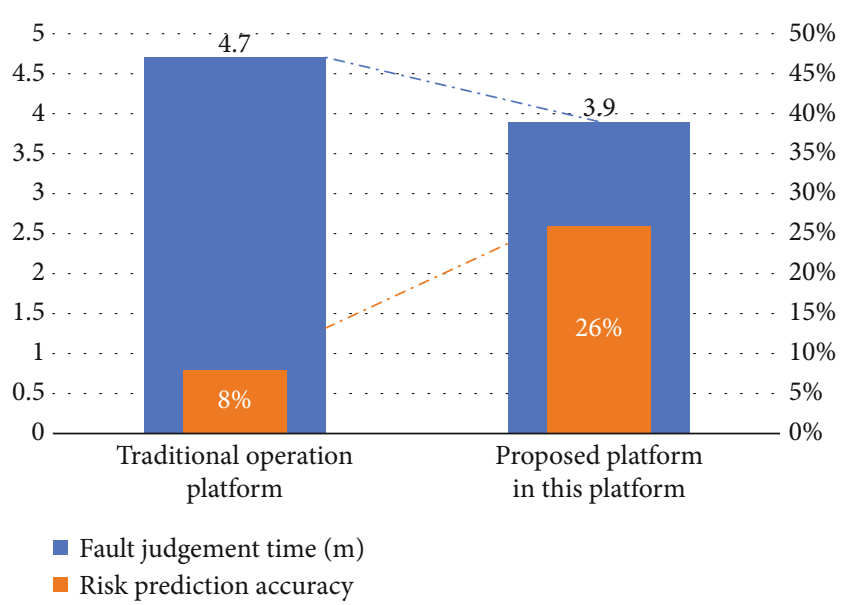

Figure 12: Comparison of fault judgment time and risk prediction accuracy.

overcomes the limitations of the traditional network management only supporting the operation and maintenance team.

6.2. Comparison with Traditional Alarm Analysis. To improve the accuracy and timeliness of the alarm impact and risk judgment for the operation and maintenance support team, in this system, it displays not only the alarm information but also the alarm and the related risk assessment of the alarm. Figure 12 shows the comparison of fault judgment time and risk prediction accuracy between the traditional operation platform and the proposed platform in this paper.

As shown in Figure 12, compared with the traditional alarm analysis that relies on manual analysis of historical alarms, the experiment to design operation and maintenance system integrating the Apriori association rule for the telecom platform in this paper effectively improves the judgment efficiency of the operation and maintenance support team on the cause of the fault. Thus, the time of risk determination reduced from 4.7 minutes to 3.9 minutes. At the same time, it also significantly improved the accuracy of risk prediction, and the risk prediction increased from $8 \%$ to $26 \%$.

\section{Conclusions and Future Work}

In this paper, we have followed the experiment of operation and maintenance system integrating the Apriori algorithm to discover the association rules in large-scale data and we have developed an operation and maintenance system integrating the Apriori association rule for the telecom platform via designing the system architecture, database, front end, and back end. The experimental results show that the system can effectively overcome the limitations of the architecture, function, and user collaboration mode of the traditional operation platform and improve the judgment efficiency of the fault cause for the operation and maintenance support team and the accuracy of risk prediction.

Compared with the current operation and maintenance system of the telecom platform, although this work has certain characteristics and advantages, it still has many short- comings due to the limitation of development time and computing resources. As a future work, we are planning to improve the existing work from the following aspects:

(1) Mobile-end security. The operation and access of the system are mainly achieved via the network; although the system has done security design for the user access rights, there are still some security problems; e.g., it is possible to bypass user authentication for the collaboration features of the social network. In the future, we will introduce the more robust authentication mechanism of the mobile end to overcome the security risks

(2) Multiplatform adaptability. This work suffers from a lack of data acquisition capacity for the non-Linux/ UNIX server's platform and external network management system, and the multiplatform adaptability is weak. In the future, we will enhance the capacity of data acquisition and improve the external interface functions

(3) Fault risk assessment. Although this work has improved the accuracy of fault risk prediction affecting the business level, there is also misjudgement of the risk level in actual operation. In the future, we will continuously optimize the evaluation model based on the application experience and further reduce the probability of alarm misjudgement

(4) Model construction of predictive and prescriptive maintenance. This work mainly uses Apriori association rules to conduct a preliminary experiment of the operation and maintenance system for the telecom platform, so in the experimental design and test, there is not much involved in model prediction and system standardization and other factors. To some extent, it determines the generality and portability of the built model

In addition, we would also like to continue experimenting with other implementation techniques based on the telecom platform and others specific for fixed-end and mobile-end devices.

\section{Data Availability}

Data sharing is not applicable to this article as no datasets were generated or analysed during the current study.

\section{Conflicts of Interest}

The authors declare that they have no conflicts of interest.

\section{Acknowledgments}

This work was partially supported by the Science and Technology Commission of Shanghai Municipality (No. 19142201600) in China and the Graduate Innovation and Entrepreneurship Program in Shanghai University in China (No. 2019GY04). 


\section{References}

[1] J. Mulongo, M. Atemkeng, T. Ansah-Narh, R. Rockefeller, G. M. Nguegnang, and M. A. Garuti, "Anomaly detection in power generation plants using machine learning and neural networks," Applied Artificial Intelligence, vol. 34, no. 1, pp. 64-79, 2020.

[2] M. Sorrentino, V. Cirillo, D. Panagrosso, A. Trifiro, and F. Bedogni, "Development of free-cooling detection procedures to support energy intelligence actions within telecommunication environments," Applied Thermal Engineering, vol. 144, pp. 1037-1048, 2018.

[3] S. Van Rossem, W. Tavernier, D. Colle, M. Pickavet, and P. Demeester, "Introducing development features for virtualized network services," IEEE Communications Magazine, vol. 56, no. 8, pp. 184-192, 2018.

[4] C. Heracleous, P. Kolios, C. G. Panayiotou, G. Ellinas, and M. M. Polycarpou, "Hybrid systems modeling for critical infrastructures interdependency analysis," Reliability Engineering and System Safety, vol. 165, pp. 89-101, 2017.

[5] R. Kaur, V. Krishnasamy, and N. K. Kandasamy, "Optimal sizing of wind-PV-based DC microgrid for telecom power supply in remote areas," IET Renewable Power Generation, vol. 12, no. 7, pp. 859-866, 2018.

[6] R. Kaur, V. Krishnasamy, K. Muthusamy, and P. Chinnamuthan, "A novel proton exchange membrane fuel cell based power conversion system for telecom supply with genetic algorithm assisted intelligent interfacing converter," Energy Conversion and Management, vol. 136, pp. 173-183, 2017.

[7] X. Chen, "Optimizing MPBSM resource allocation based on revenue management: a China mobile Sichuan case," Mobile Information Systems, vol. 2015, Article ID 892705, 10 pages, 2015.

[8] L. Bennacer, Y. Amirat, A. Chibani, A. Mellouk, and L. Ciavaglia, "Self-diagnosis technique for virtual private networks combining Bayesian networks and case-based reasoning," IEEE Transactions on Automation Science and Engineering, vol. 12, no. 1, pp. 354-366, 2015.

[9] N. Lu, G. Q. Zhang, and J. Lu, "Concept drift detection via competence models," Artificial Intelligence, vol. 209, pp. 11$28,2014$.

[10] C. Lozano-Garzon, C. Ariza-Porras, S. Rivera-Diaz, H. Riveros-Ardila, and Y. Donoso, "Mobile network QoEQoS decision making tool for performance optimization in critical web service," International Journal of Computers Communications and Control, vol. 7, no. 5, pp. 892-899, 2014.

[11] Y. S. Rong and C. F. Li, "Design of integrated operation and maintenance support system of telecommunication platform based on web and light app," Electronic Technology and Software Engineering, vol. 20, no. 1, pp. 46-47, 2015.

[12] X. J. Geng, Y. Liang, and L. M. Jiao, "EARC: evidential association rule-based classification," Information Sciences, vol. 547, pp. 202-222, 2021.

[13] M. S. Bashkari, A. Sami, and M. Rastegar, "Outage cause detection in power distribution systems based on data mining," IEEE Transactions on Industrial Informatics, vol. 17, no. 1, pp. 640-649, 2021.

[14] Y. H. He, Y. X. Zhao, X. Han, D. Zhou, and W. Z. Wang, "Functional risk-oriented health prognosis approach for intelligent manufacturing systems," Reliability Engineering \& System Safety, vol. 203, article 107090, 2020.
[15] Q. Jiang, Z. X. Xiao, C. P. Ru et al., "Study on the modeling method of knowledge base system in web environment," International Journal of Pattern Recognition and Artificial Intelligence, vol. 33, no. 9, article 1959031, 2019.

[16] S. Wan, D. B. Li, J. Gao, and J. Li, “A knowledge based machine tool maintenance planning system using case-based reasoning techniques," Robotics and Computer-Integrated Manufacturing, vol. 58, pp. 80-96, 2019.

[17] N. Hai, D. Q. Gong, and S. F. Liu, "Ontology knowledge base combined with Bayesian networks for integrated corridor risk warning," Computer Communications, vol. 174, pp. 190-204, 2021.

[18] J. Y. Jhang, I. S. Tzeng, H. H. Chou et al., "Association rule mining and prognostic stratification of 2-year longevity in octogenarians undergoing endovascular therapy for lower extremity arterial disease: observational cohort study," Journal of Medical Internet Research, vol. 22, no. 12, article e17487, 2020.

[19] J. Y. Liao, S. Wu, and A. L. Liu, "High utility itemsets mining based on divide-and-conquer strategy," Wireless Personal Communications, vol. 116, no. 3, pp. 1639-1657, 2021.

[20] D. Y. Li and Y. L. Liao, "Pollution zone identification research during ozone pollution processes," Environmental Monitoring and Assessment, vol. 192, no. 9, p. 591, 2020.

[21] M. Al-Fayoumi, J. Alwidian, and M. Abusaif, "Intelligent association classification technique for phishing website detection," International Arab Journal of Information Technology, vol. 17, no. 4, pp. 488-496, 2020.

[22] F. Safara, A. Souri, and M. Serrizadeh, "Improved intrusion detection method for communication networks using association rule mining and artificial neural networks," IET Communications, vol. 14, no. 7, pp. 1192-1197, 2020.

[23] S. Raj, D. Ramesh, M. Sreenu, and K. K. Sethi, "EAFIM: efficient Apriori-based frequent itemset mining algorithm on spark for big transactional data," Knowledge and Information Systems, vol. 62, no. 9, pp. 3565-3583, 2020.

[24] B. Shazad, H. U. Khan, M. Zahoor-ur-Rehman et al., "Finding temporal influential users in social media using association rule learning," Intelligence Automation and Soft Computing, vol. 26, no. 1, pp. 87-98, 2019.

[25] L. J. Chen, K. Mao, Y. Zheng, X. Zhou, and C. M. Zhu, "Research on mining association rules in university scientific projects management," Network Computing and Information Security, vol. 345, no. 1, pp. 561-567, 2012.

[26] Y. Xu, M. M. Zeng, Q. Liu, and X. Wang, "A genetic algorithm based multilevel association rules mining for big datasets," Mathematical Problems in Engineering, vol. 2014, no. 12, Article ID 867149, p. 9, 2014.

[27] L. H. Ding, S. S. Xie, S. C. Zhang et al., "Delayed comparison and Apriori algorithm (DCAA): a tool for discovering protein-protein interactions from time-series phosphoproteomic data," Frontiers in Molecular Biosciences, vol. 7, article 606570, 2020.

[28] Z. He and J. H. Chen, "Research on service innovation methods of service-oriented manufacturing enterprises based on product-service integration," Operations Research and Management Science, vol. 24, no. 3, pp. 240-247, 2015.

[29] D. M. Cui, "Empirical analysis of statistical data mining in big data era," Statistics and Decision, vol. 48, no. 4, pp. 180-182, 2016. 
[30] Y. H. Lin and C. F. Chen, "Research on enterprise financial risk evaluation based on association rules," Friends of Accounting, vol. 16, no. 1, pp. 32-35, 2017.

[31] O. F. Althuwaynee, A. Aydda, I. T. Hwang et al., "Uncertainty reduction of unlabeled features in landslide inventory using machine learning t-SNE clustering and data mining Apriori association rule algorithms," Applied Sciences-Basel, vol. 11, no. 2, p. 556, 2021.

[32] X. G. Guo and Y. P. Fan, "Research on tax evasion identification path of tax system based on big data," Mathematics in Practice and Theory, vol. 48, no. 3, pp. 92-100, 2018.

[33] T. J. Li and D. W. Yan, "Research on Weibo recommendation based on emotional weighted association rules," Data Analysis and Knowledge Discovery, vol. 4, no. 4, pp. 27-33, 2020.

[34] B. Y. Chen, J. Ding, and S. N. Chen, "Selection of key incentives for power production safety accidents based on association rule mining," Electric Power Automation Equipment, vol. 38, no. 4, pp. 68-74, 2018.

[35] D. Tian, Y. Shen, M. C. Li, and S. Han, "An intelligent data mining approach of text entity knowledge from construction documents of concrete dam," Journal of Hydroelectric Engineering, vol. 40, no. 6, pp. 139-151, 2021.

[36] L. Q. You, X. X. Li, Y. Q. Li, F. Li, and D. Liu, "Correlation analysis of cigarette physical indexes based on Apriori association rules," Light Industry Science and Technology, vol. 37, no. 3, pp. 88-92, 2021.

[37] F. Li, J. L. Guo, and L. M. Tan, "Association analysis of company shareholders based on Apriori algorithm," Software Guide, vol. 20, no. 3, pp. 106-109, 2021.

[38] W. J. Cui, X. N. Ma, and S. Q. Sun, "Railway network security early warning method based on improved Apriori algorithm," Railway Computer Application, vol. 30, no. 3, pp. 59-64, 2021.

[39] Y. Q. Zhang, H. Q. Wang, S. Z. Wang, and L. Q. Gao, "Mining method for parking space idle pattern based on Hadoop," Journal of Tianjin Normal University (Natural Science Edition), vol. 41, no. 1, pp. 69-74, 2021.

[40] J. W. Xia, B. X. Liu, and C. F. Luo, "Analysis of mobile APP and advertising investment based on Apriori algorithm," China Management Information, vol. 24, no. 2, pp. 62-64, 2021.

[41] R. N. Yang, Research and software realization of remote supervision and control system based on web, Tianjin University of Technology Master's Degree, 2008.

[42] H. A. Kang, "Developing mobile apps in HTML5," Science and Technology Innovation Herald, vol. 7, pp. 30-31, 2012.

[43] X. Qiao, J. Chen, P. Gu, and Y. Li, "Opening up telecom networks with a lightweight web element service cloud for ordinary users in the web 2.0 era," IEEE Communications Magazine, vol. 52, no. 10, pp. 127-133, 2014.

[44] C. Borgelt and R. Kruse, "Induction of association rules: Apriori implementation," Leukemia Research, vol. 8, no. 6, pp. 937-944, 1984.

[45] R. Perego, S. Orlando, and P. Palmerini, "Enhancing the Apriori algorithm for frequent set counting," in Data Warehousing and Knowledge Discovery, vol. 2114, pp. 71-82, Springer, Berlin, Heidelberg, 2001.

[46] Y. Z. Wu, "Design and implementation of improved Apriori algorithm in telecommunication alarm system," Computer Knowledge and Technology, vol. 7, no. 36, pp. 9423-94237, 2011.

[47] Y. Huang, Research and application of association rule analysis in telecommunication warning system, University of Electronic Science and Technology of China Master's Degree, 2007. 